\title{
ALMOST-FINITE, COMPACT, AND INESSENTIAL OPERATORS
}

\section{DAVID KLEINECKE}

We are concerned here with four closely related ideals in the Banach algebra of endomorphisms of a Banach space (cf. [1, pp. $51 \mathrm{ff}$.]). The term operator will be restricted to members of this algebra. Ideals are assumed to be two-sided.

The most tractable operators, in the sense of actual computation, are those whose range is spanned by a finite set of elements. Such operators can be called, briefly, finite operators (the term degenerate operator is also used). The finite operators form an ideal which is not closed in any conventional topology. The other three ideals mentioned above are closed extensions of the ideal of finite operators.

An operator will be called almost-finite if it is the uniform limit of a sequence of finite operators.

An operator will be called compact if it takes bounded sets into sets with a compact closure. The term completely continuous operator is also used for these operators.

An operator will be called inessential if its image in the quotient algebra of the algebra of all operators over the almost-finite operators belongs to the (Jacobson) radical. For a definition of the radical see [1, Chapter 24], or below in the proof of Theorem 1.

It follows at once from these definitions that the sets of almostfinite, compact, and inessential operators are all ideals. Moreover, each of these ideals is closed; the first by definition, the second by a well known theorem (cf. [1, p. 49]), and the third because the radical of a Banach algebra is closed and the mapping into a quotient algebra is continuous.

It is well known that almost-finite operators are compact (cf. [1, p. 49]). In many cases these two ideals are equal; however, it is still an open question whether or not this is true in general. It is an immediate consequence of Theorem 1 that compact operators are inessential. In some spaces (for example, separable Hilbert space which has only one nontrivial closed ideal) inessential operators are compact; there are others in which they are not equal (cf. the introduction of [2]), but this question does not appear to have been investigated in detail.

Presented to the Society, September 3, 1954 under the title $A$ generalization of complete continuity; received by the editors July 23, 1962 and, in revised form, August $27,1962$. 
The quotient algebra of the algebra of all operators over any of these closed ideals forms a Banach algebra under the usual quotient norm (cf. [1, pp. $697 \mathrm{ff}$.]). The quotient algebras over the almostfinite, compact, and inessential operators can be called the suprafinite, supra-compact, and essential algebras, respectively. Since the kernel ideals are ordered by inclusion these quotient algebras form a chain of homomorphic images. Note that, since the essential algebra is semi-simple (i.e. has a trivial radical), the process leading from the almost-finite operators to the essential operators cannot be repeated to yield further generalizations.

An isolated spectral point is of finite multiplicity if there is a finite idempotent which commutes with the operator such that the point in question is not in the spectrum of the operator restricted to the range of the complementary idempotent; all such spectral points are necessarily eigenvalues (cf. [1, pp. $177 \mathrm{ff}$.]). An operator will be described as having a Riesz spectrum if its nonzero spectrum consists of a set (possibly empty) of isolated eigenvalues of finite multiplicity.

It is well known that almost-finite and compact operators have Riesz spectra (cf. [1, pp. $180 \mathrm{ff}$.]). We will prove (Theorem 8 ) that inessential operators have Riesz spectra; moreover, the ideal of inessential operators is maximal in this respect as is shown in the following theorem.

THEOREM 1. Every ideal of operators with Riesz spectra is included in the inessential operators.

Proof. Suppose $X$ is a member of an ideal of operators with Riesz spectra; then, for any $Y$, both $X Y$ and $Y X$ have Riesz spectra. Suppose $\lambda$ belongs to the resolvent set of $X Y$; since inverses are preserved under homomorphisms $\lambda$ is in the resolvent set of $x y$, the image of $X Y$ in the supra-finite algebra. On the other hand, if $\lambda \neq 0$ is in the spectrum of $X Y$ there is a finite idempotent $P$ such that $\lambda$ is not in the spectrum of $X Y$ restricted to the range of $I-P$. In other words, there are operators $U$ and $V$ such that

$$
U(\lambda-(I-P) X Y)=I-P=(\lambda-(I-P) X Y) V ;
$$

and, therefore

$$
\begin{aligned}
& U(\lambda-X Y)=I-(P+U P X Y), \\
& (\lambda-X Y) V=I-(P+P X Y V) .
\end{aligned}
$$

But $P+U P X Y$ and $P+P X Y V$ are finite; hence $U$ and $V$ map into left and right inverses of $\lambda-x y$, and $\lambda$ is in the resolvent set of $x y$. Thus $x y$ is quasi-nilpotent; in the same way, $y x$ is quasi-nilpotent. The 
radical of the supra-finite algebra is, by definition, the set of all $x$ such that, for every $y, x y$ and $y x$ are quasi-nilpotent. Thus $x$ is in the radical and $X$ is inessential.

It should be pointed out that an ideal of operators with Riesz spectra is no more restrictive than an ideal of operators which have finite multiplicity (including possibly zero) at some fixed point (say 1 ) in the spectral plane. This follows because $\lambda^{-1} R$ will have finite multiplicity for all $\lambda \neq 0$ and, since $(\lambda-R)^{-1}=\lambda^{-1}\left(1-\lambda^{-1} R\right)^{-1}, R$ will have finite multiplicity at all $\lambda \neq 0$.

An operator will be called essentially regular if its image in the essential algebra has an inverse. Essential regularity can be expressed in terms of finite operators as is shown in the following theorem.

TheоRem 2. The following statements are equivalent:

(a) $X$ is essentially regular;

(b) the image of $X$ in the supra-finite algebra has an inverse;

(c) there are operators $U$ and $V$ such that $I-X U$ and $I-V X$ are finite.

Proof. (a) and (b) are equivalent by a standard algebraic theorem (cf. [1, p. 697]). If (c) holds, (b) is obvious. If (b) holds, there is an operator $T$ such that

$$
X T=I-F \quad \text { and } \quad T X=I-G,
$$

where $F$ and $G$ are almost-finite operators. Since $F$ is almost-finite there is at least one finite operator $H$ such that $\|F-H\|<1$; then $(I-(F-H))^{-1}$ exists and

$$
I-X T(I-(F-H))^{-1}=H(I-(F-B))^{-1}
$$

which is finite. The second half of (c) follows in the same way.

The calculus of deficiency indices is useful in this context. For any operator $X$, let $\nu X$ be the maximum number of linearly independent elements taken into zero by $X$, and let $\mu X=\nu X^{*}$ where $X^{*}$ is the adjoint operator corresponding to $X$. If the range of $X$ is closed $\mu X$ is the maximum number of linearly independent elements not in the range of $X$.

For a detailed exposition of results concerning deficiency indices see [3]; we will need the following three theorems which we state without proof.

THEOREM 3. $X$ is essentially regular if and only if the range of $X$ is closed, $\nu X<\infty$ and $\mu X<\infty$. 
THEOREM 4. If $X$ and $Y$ are essentially regular then $X Y$ is essentially regular and

$$
\mu(X Y)-\mu X-\mu Y=\nu(X Y)-\nu X-\nu Y .
$$

THEOREM 5. If $X$ is essentially regular then there is a constant $\eta>0$ such that, for all essentially regular $Y$ for which $\|X-Y\|<\eta$,

$$
\mu X-\mu Y=\nu X-\nu Y .
$$

The deficiency indices are not sensitive to the addition of an inessential operator as is shown in the following theorem.

Theorem 6. If $X$ is essentially regular and $R$ is inessential then $X+R$ is essentially regular and

$$
\mu(X+R)-\mu X=\nu(X+R)-\nu X .
$$

Proof. If $X$ is essentially regular then its image in the essential algebra has an inverse. The image of $X+\lambda R$, for any scalar $\lambda$, is the same as the image of $X$ and, therefore, has an inverse. Thus $X+\lambda R$, and in particular $X+R$, is essentially regular. Now, by Theorem 5, there is a neighborhood of every $\lambda$ such that

$$
\mu\left(X+\lambda^{\prime} R\right)-\mu(X+\lambda R)=\nu\left(X+\lambda^{\prime} R\right)-\nu(X+\lambda R)
$$

for all $\lambda^{\prime}$ in the neighborhood. Consequently, $\mu(X+\lambda R)-\nu(X+\lambda R)$ is independent of $\lambda$; hence

$$
\mu(X+R)-\mu X=\nu(X+R)-\nu X .
$$

The following theorem is particularly useful in some perturbation problems.

THEOREM 7 (NIKOL'SKII''s THEOREM, cf. [4]). If $X+R$ has an inverse and $R$ is inessential then there is a finite operator $F$ such that $X+F$ has an inverse.

Proof. Since $X+R$ has an inverse it is essentially regular, and moreover, $\mu(X+R)=\nu(X+R)=0$. Hence, by Theorem $6, X$ is essentially regular and

$$
\mu X-\nu X=\mu(X+R)-\nu(X+R)=0 .
$$

The remainder of the proof requires that a finite operator $F$ be constructed such that $X+F$ has an inverse. Suppose that $\psi_{1}, \psi_{2} \cdots \psi_{m}$ are a maximal set of linearly independent elements not in the range of $X$ and that $\phi_{1}, \phi_{2} \cdots \phi_{n}$ are a maximal set of linearly independent elements taken into zero by $X$. A set of functionals $\phi_{1}^{*}, \phi_{2}^{*} \cdots \phi_{n}^{*}$ can be found by the Hahn-Banach theorem such that $\phi_{i}^{*} \phi_{i}=1$ and 
$\phi_{i}{ }^{*} \phi_{j}=0$ if $i \neq j$. Since $\mu X=\nu X$ it follows that $n=m$ so that the $\phi_{i}^{*}$ and $\psi_{i}$ can be placed in one-to-one correspondence. Now let

$$
F \theta=\sum_{i=1}^{n} \phi_{i}^{*} \theta \cdot \psi_{i}
$$

which defines a finite operator $F$. Now suppose $(X+F) \theta=0$, then

$$
0=X \theta+\sum_{i=1}^{n} \phi_{i}^{*} \theta \cdot \psi_{i}
$$

so that, by the definition of the $\psi_{i}, 0=X \theta=\phi_{i}^{*} \theta$ and, therefore, $\theta=0$. Furthermore if $\psi$ is any element then

$$
\psi=X \theta+\sum_{i=1}^{n} \alpha_{i} \psi_{i}
$$

for some element $\theta$ and some scalars $\alpha_{i} ; \theta$ can be chosen so that $\phi_{i}^{*} \theta=0$ for all $i$. Hence

$$
(X+F)\left(\theta+\sum_{i=1}^{n} \alpha_{i} \phi_{i}\right)=X \theta+\sum_{i=1}^{n} \alpha_{i} \psi_{i}=\psi,
$$

so that $X+F$ is one-to-one onto and, therefore, has an inverse.

We have said almost nothing so far about compact operators. One of the principal points of this paper is that there is very little to say which is not better said concerning inessential operators. It seems that most known theorems about compact operators generalize directly, as did, for example, Theorem 7. We will, however, limit ourselves to this one example.

Finally we show that inessential operators have a Riesz spectrum.

Theorem 8. If $R$ is inessential then $R$ has a Riesz spectrum.

Proof. Suppose $\lambda \neq 0$, then $\lambda=(\lambda-R)+R$ has an inverse so that, by Theorem 7 , there is a finite operator $F$ such that $\lambda-R+F$ has an inverse. Hence there is an operator $U$ such that $R=U+F$ and $\lambda$ is not in the spectrum of $U$. Then by a known perturbation theorem, $U+F$ can have only isolated eigenvalues of finite multiplicity in a neighborhood of $\lambda$. Since this holds for arbitrary $\lambda \neq 0, R$ has a Riesz spectrum.

The referee has pointed out that most of these results generalize immediately to more general linear topological spaces, in particular to algebras without unit, provided the finite operators are replaced by some arbitrary ideal (not necessarily closed). The results may well provide a useful tool in more general spaces but we have retained 
the formulation given above because the finite operators do play such a distinguished role in applications of the theory.

\section{REFERENCES}

1. E. Hille and R. S. Phillips, Functional analysis and semi-groups, Amer. Math. Soc. Colloq. Publ. Vol. 31, rev. ed., Amer. Math. Soc., Providence, R. I., 1957.

2. B. Yood, Difference algebras of linear transformations on a Banach space, Pacific J. Math. 4 (1954), 615-636.

3. I. C. Gohberg and M. G. KreYn, Fundamental aspects of defect numbers, root numbers and indices of linear operators, Uspehi Mat. Nauk 12 (1957), no. 2(74), 43-118. (Russian)

4. S. M. Nikol'skir, Linear equations in normed linear spaces, Izv. Akad. Nauk SSSR Ser. Mat. 7 (1943), 147-166. (Russian)

General Electric Company, Tempo, Santa Barbara, California 